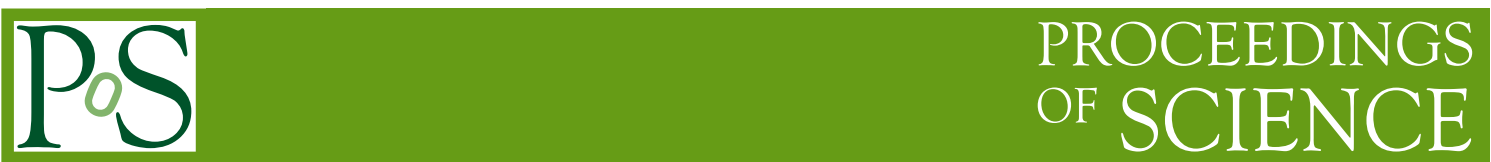

\title{
Experimental overview on $D_{(s)}$ purely leptonic decays
}

\author{
Hailong Ma*† \\ Institute of High Energy Physics, Chinese Academy of Sciences \\ E-mail: mahleihep.ac.cn
}

Up to now, much progress in the studies of the purely leptonic decays $D_{(s)}^{+} \rightarrow \ell^{+} v_{\ell}(\ell=e, \mu$ or $\tau)$ has been reached in various experiments. This proceeding overviews the experimental studies of $D_{(s)}^{+} \rightarrow \ell^{+} v_{\ell}$ at the CLEO-c, Belle, BaBar and BESIII experiments in recent ten years.

9th International Workshop on the CKM Unitarity Triangle

28 November - 3 December 2016

Tata Institute for Fundamental Research (TIFR), Mumbai, India

\footnotetext{
${ }^{*}$ Speaker.

${ }^{\dagger}$ I would like to thank for the support of the National Natural Science Foundation of China (NSFC) under Contract No. 11305180 and the Ministry of Science and Technology of China (973 by MOST) under Contract 2009CB825200.
} 


\section{Introduction}

In the Standard Model (SM) of particle physics, the $D_{(s)}^{+}$(through this proceeding, charge conjugation is implied) meson can decay into $\ell^{+} v_{\ell}$ (where $\ell$ is $e, \mu$ or $\tau$ ) through a virtual $W^{+}$ boson. The decay rate. To the lowest order, the decay width of $D_{(s)}^{+} \rightarrow \ell^{+} v_{\ell}$ can be simply written as

$$
\Gamma\left(D_{(s)}^{+} \rightarrow \ell^{+} v_{\ell}\right)=\frac{G_{F}^{2} f_{D_{(s)}^{+}}^{2}}{8 \pi}\left|V_{c d(s)}\right|^{2} m_{\ell}^{2} m_{D_{(s)}^{+}}\left(1-\frac{m_{\ell}^{2}}{m_{D_{(s)}^{+}}^{2}}\right)^{2},
$$

where $G_{F}$ is the Fermi coupling constant, $f_{D_{(s)}^{+}}$is the decay constant of $D_{(s)}^{+},\left|V_{c d(s)}\right|$ is the CabibboKobayashi-Maskawa (CKM) matrix element [1] in $D_{(s)}^{+}, m_{l}$ and $m_{D_{(s)}^{+}}$are the masses of lepton and $D_{(s)}^{+}$. With the accurate CKM matrix element $\left|V_{c d(s)}\right|$ from the SM global fit, one can determine $f_{D_{(s)}^{+}}$ and verify the LQCD calculations on these parameters. In recent years, the LQCD calculations of $f_{D^{+}}$have reached a high precision of $0.5 \%[2,3,4]$. With the precisely measured branching fraction of $D_{(s)}^{+} \rightarrow \ell^{+} v_{\ell}$ and the precisely calculated $f_{D^{+}}$by LQCD, one can extract $\left|V_{c d(s)}\right|$ with improved precision, thus testing the CKM matrix unitary with better precision.

Experimental studies of $D^{+} \rightarrow \ell^{+} v_{\ell}$ have mainly come from $e^{+} e^{-}$experiments near $D^{+} D^{-}$ production threshold, which are MARKIII [5], BESI [6], BESII [7], CLEO-c [8, 9, 10] and BESIII [11]. These experiments used the datasets taken around $3.773 \mathrm{GeV}$, except for the BESI experiment at $4.03 \mathrm{GeV}$. Meanwhile, experimental studies of $D_{s}^{+} \rightarrow \ell^{+} v_{\ell}$ have been performed by the fixed-target experiments (CERN WA75 [12] Fermilab E653 [13] and CERN WA92 [14]); using $Z^{0}$ decays at $91 \mathrm{GeV}$ (L3 [15], OPAL [16] and ALEPH [17]); using $B$ decays produced around $\Upsilon(4 S)$ and $\Upsilon(5 S)$ (CLEO [18], Belle [19, 20] and BaBar [21]) and using $D_{s}^{+} D_{s}^{-}\left(D_{s}^{+} D_{s}^{*-}\right.$ ) meson pairs accumulated at 4.03 (4.14), 4.17 and $4.009 \mathrm{GeV}$ (BESI [22], CLEO [23, 24, 25] and BESIII [26]. Herein, we mainly overview the studies of $D^{+} \rightarrow \ell^{+} v_{\ell}$ at the CLEO-c [10] and BESIII [11] experiments, and those of $D_{s}^{+} \rightarrow \ell^{+} v_{\ell}$ at the CLEO-c [23, 24, 25], Belle [20], BaBar [21] and BESIII [26] experiments in recent ten years.

\section{2. $D^{+}$leptonic decays}

\subsection{Measurement of $D^{+} \rightarrow \ell^{+} v_{\ell}$ at CLEO-c}

In 2008, the CLEO collaboration reported analysis of $D^{+} \rightarrow \ell^{+} v_{\ell}$ by using $0.818 \mathrm{fb}^{-1}$ dataset taken around $3.774 \mathrm{GeV}[10]$. At this energy, $D^{+}$and $D^{-}$mesons are produced in pair. One can measure absolute branching fraction with clean $D^{+}$sample using the well established doubletag method. They accumulated singly tagged $460055 \pm 787 D^{-}$mesons with 6 hadronic decays $\left(D^{-} \rightarrow K^{+} \pi^{-} \pi^{-}\left(\pi^{0}\right), K_{S}^{0} \pi^{-}\left(\pi^{0}\right), K^{+} K^{-} \pi^{-}\right.$and $\left.K_{S}^{0} \pi^{+} \pi^{-} \pi^{-}\right)$. In the recoil sides of these tagged $D^{-}$mesons, they obtained $149.7 \pm 12.0$ signal events of $D^{+} \rightarrow \ell^{+} v_{\ell}(\ell=\mu$ or $\tau)$, where the $\mu^{+} v_{v}$ and $\tau^{+} v_{\tau}$ yields have been constrained by the SM prediction. They determined $B\left(D^{+} \rightarrow \mu^{+} v\right)=$ $(3.82 \pm 0.32 \pm 0.09) \times 10^{-4}$ and $f_{D^{+}}=(205.8 \pm 8.5 \pm 2.5) \mathrm{MeV}$. Without this constraint, they obtained $B\left(D^{+} \rightarrow \mu^{+} v\right)=(3.93 \pm 0.35 \pm 0.09) \times 10^{-4}, f_{D^{+}}=(207.6 \pm 9.3 \pm 2.5) \mathrm{MeV}$ and an upper limit of $B\left(D^{+} \rightarrow \tau^{+} v_{\tau}\right)<1.2 \times 10^{-3}$ at $90 \%$ C.L.. 


\subsection{Improved measurement of $D^{+} \rightarrow \mu^{+} v_{\mu}$ at BESIII}

During 2010 and 2011, a dateset of $2.93 \mathrm{fb}^{-1}$ was collected around $3.773 \mathrm{GeV}$ with the BESIII detector. From this dataset, BESIII accumulated $(170.31 \pm 0.34) \times 10^{4}$ singly tagged $D^{-}$mesons with 9 hadronic decays $D^{-} \rightarrow K^{+} \pi^{-} \pi^{-}\left(\pi^{0}\right), K_{S}^{0} \pi^{-}\left(\pi^{0}\right), K^{+} K^{-} \pi^{-}, K_{S}^{0} \pi^{+} \pi^{-} \pi^{-}, K_{S}^{0} K^{-}, \pi^{+} \pi^{-} \pi^{-}$ and $K^{+} \pi^{+} \pi^{-} \pi^{-} \pi^{-}$, with the fitted beam-constrained mass (MC $\left.M_{\mathrm{BC}}\right)$ spectra shown in Fig. 1 (left). Figure 1 (right) shows the $M_{\text {miss }}^{2}$ (the missing mass square of the $D^{-} \mu^{+}$system) distribution of the $D^{+} \rightarrow \mu^{+} v_{\mu}$ candidates, which are selected selected with the muon counter in the systems against the tagged $D^{-}$mesons. BESIII obtained $409 \pm 21 D^{+} \rightarrow \mu^{+} v_{\mu}$ signals after background subtraction, which leads to $\mathscr{B}\left(D^{+} \rightarrow \mu^{+} v_{\mu}\right)=\left(3.71 \pm 0.19_{\text {stat. }} \pm 0.06_{\text {sys. }}\right) \times 10^{-4}, f_{D^{+}}=203.2 \pm$ $5.3_{\text {stat. }} \pm 1.8_{\text {sys. }} \mathrm{MeV}$ and $\left|V_{c d}\right|=0.2210 \pm 0.058_{\text {stat. }} \pm 0.047_{\text {sys. }}$, respectively [11].
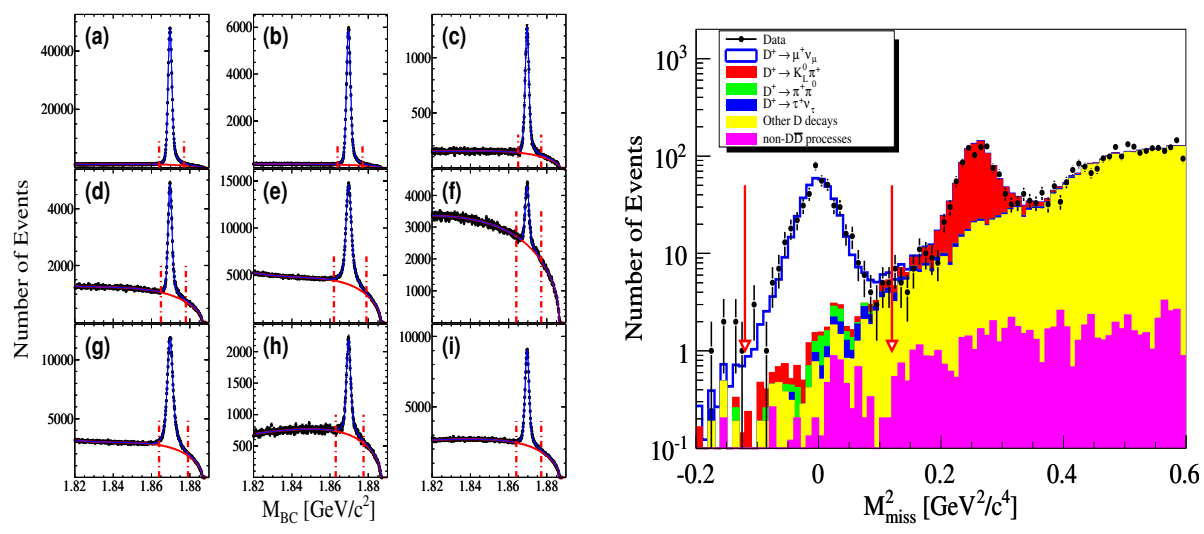

Figure 1: (Left) Fits to the $M_{\mathrm{BC}}$ spectra for the singly tagged $D_{s}^{-}$candidates, where (a-f) can be found in Ref. [11]. (Right) $M_{\text {miss }}^{2}$ distribution of the $D^{+} \rightarrow \mu^{+} v_{\mu}$ candidates.

\subsection{Preliminary result of the evidence for $D^{+} \rightarrow \tau^{+} v_{\tau}$ at BESIII}

Using the same dataset, BESIII report the preliminary result on search for $D^{+} \rightarrow \tau^{+}\left(\pi^{+} \bar{v}_{\tau}\right) v_{\tau}$. In this analysis, 6 hadronic decays $D^{-} \rightarrow K^{+} \pi^{-} \pi^{-}\left(\pi^{0}\right), K_{S}^{0} \pi^{-}\left(\pi^{0}\right), K^{+} K^{-} \pi^{-}$and $K_{S}^{0} \pi^{+} \pi^{-} \pi^{-}$ are used to reconstruct singly tagged $D^{-}$mesons. No particle identification is performed to select the pion candidate of the signal side. To reject the backgrounds of $D^{+} \rightarrow \pi^{+} K_{L}^{0}$ and $D^{+} \rightarrow \pi^{+} \pi^{0}$ in the signal regions, the missing momentum representing the neutrino(s) of a signal event should be not in the beam direction, and the most energetic neutral EMC shower should not be aligned with the missing momentum. Figure 2 shows the $M_{\text {miss }}^{2}$ distributions of the accepted candidates for $D^{+} \rightarrow \tau^{+} v_{\tau}$, which have been categorized into $\mu$-like $\left(E_{\mathrm{EMC}}<300 \mathrm{MeV}\right)$ and $\pi$-like $\left(E_{\mathrm{EMC}}>300\right.$ $\mathrm{MeV}$ ), where $E_{\mathrm{EMC}}$ is the energy deposited in the EMC of the pion candidate. By fitting to these distributions, $137 \pm 27 D^{+} \rightarrow \tau^{+} v_{\tau}$ signals are obtained with statistical significance of more than $4 \sigma$. In the fits, all the signals and backgrounds are modeled by MC-simulated line-shapes. Moreover, the sizes of the backgrounds $D^{+} \rightarrow \mu^{+} v_{\mu}, D^{+} \rightarrow \pi^{+} \pi^{0}, D^{+} \rightarrow \pi^{+} \eta$ and $D^{+} \rightarrow \pi^{+} K_{S}^{0}$ are fixed based on MC simulations and the sizes of $D^{+} \rightarrow \tau^{+} v_{\tau}$, the backgrounds of $D^{+} \rightarrow \pi^{+} K_{L}^{0}$ and other processes are float. The branching fraction is determined to be $\mathscr{B}\left(D^{+} \rightarrow \tau^{+} v_{\tau}\right)=$ $\left(1.20 \pm 0.24_{\text {stat. }}\right) \times 10^{-3}$. Combining $\mathscr{B}\left(D^{+} \rightarrow \mu^{+} v_{\mu}\right)$ and $\mathscr{B}\left(D^{+} \rightarrow \tau^{+} v_{\tau}\right)$ measured at BESIII 
leads to the ratio of $\frac{\Gamma\left(D^{+} \rightarrow \tau^{+} v_{\tau}\right)}{\Gamma\left(D^{+} \rightarrow \mu^{+} v_{\mu}\right)}=3.21 \pm 0.64$, where the uncertainty does not include the systematic uncertainty in $\mathscr{B}\left(D^{+} \rightarrow \tau^{+} v_{\tau}\right)$. This ratio is consistent with the SM predicted value $2.66 \pm 0.01$ within about $0.9 \sigma$. The results in this part are reported for the first time.
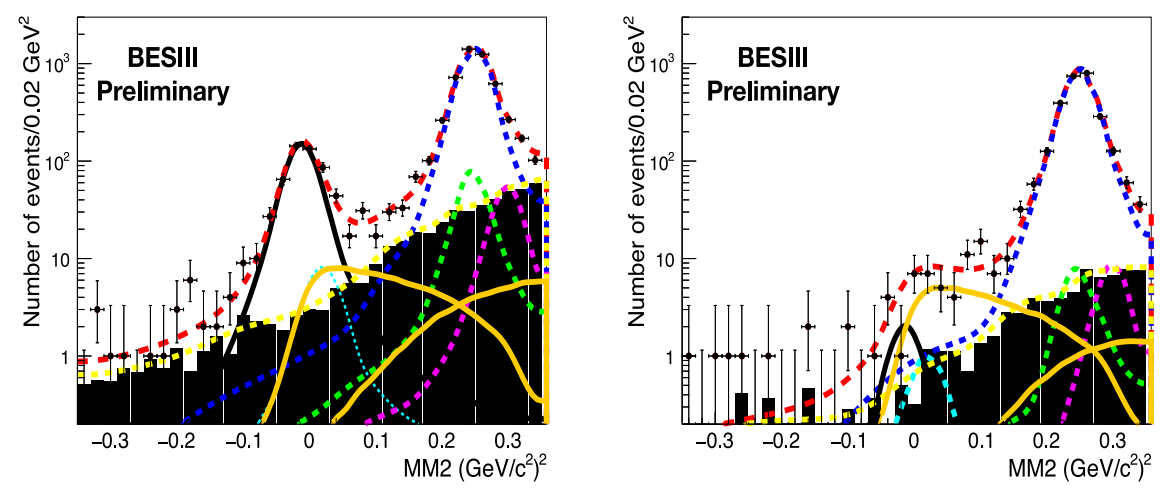

Figure 2: (Left) Fits to the $M_{\text {miss }}^{2}$ spectra of the $\mu$-like (left) and $\pi$-like (right) candidates of $D^{+} \rightarrow \tau^{+} \nu_{\tau}$. The orange and black solid curves denote the $D^{+} \rightarrow \tau^{+} v_{\tau}$ and $D^{+} \rightarrow \mu^{+} v_{\mu}$ signals. The light blue, blue, purple, green and yellow dashed curves represent the backgrounds of $D^{+} \rightarrow \pi^{+} \pi^{0}, D^{+} \rightarrow \pi^{+} K_{L}^{0}, D^{+} \rightarrow \pi^{+} \eta$, $D^{+} \rightarrow \pi^{+} K_{S}^{0}$ and other processes, respectively.

\section{3. $D_{s}^{+}$leptonic decays}

\subsection{Measurement of $D_{s}^{+} \rightarrow \ell^{+} v_{\ell}$ at CLEO-c}

In 2009, the CLEO collaboration reported the studies of $D_{s}^{+} \rightarrow \ell^{+} v_{\ell}$ with $0.6 \mathrm{fb}^{-1}$ data taken at $4.17 \mathrm{GeV}$. At this energy, $D_{s}^{+}$and $D_{s}^{-}$are produced in an asymmetrical process $e^{+} e^{-} \rightarrow D_{s}^{+} D_{s}^{*-}+$ c.c.. To study $D_{s}^{+} \rightarrow \mu^{+} v_{\mu}$ and $D_{s}^{+} \rightarrow \tau^{+}\left(\pi^{+} \bar{v}_{\tau}\right) v_{\tau}$ [23], they reconstructed the singly tagged $D_{s}^{-}$mesons using 9 hadronic decays $K^{+} K^{-} \pi^{-}\left(\pi^{0}\right), K_{S}^{0} K^{-}, \pi^{-} \eta, \pi^{-} \eta_{\pi^{+}}^{\prime} \pi^{-} \eta, \pi^{+} \pi^{-} \pi^{-}, K^{*-} K^{* 0}$, $\rho^{-} \eta$ and $\pi^{-} \eta_{\gamma \rho^{0}}^{\prime}$. They accumulated $43859 \pm 936$ singly tagged $D_{s}^{-}+\gamma$ mesons by examining the invariant mass spectra of $D_{s}^{-}$and the miss mass square of the $D_{s}^{-}+\gamma$ systems. From this sample, they obtained $235.5 \pm 13.8 D_{s}^{+} \rightarrow \mu^{+} v_{\mu}$ signals with the constraint of the ratio of the $D_{s}^{+} \rightarrow \mu^{+} v_{\mu}$ and $D_{s}^{+} \rightarrow \tau^{+} v_{\tau}$ signals. These lead to $\mathscr{B}\left(D_{s}^{+} \rightarrow \mu^{+} v\right)=\left(0.591 \pm 0.037_{\text {stat. }} \pm 0.018_{\text {sys. }}\right) \%$ and $f_{D_{s}^{+}}=\left(263.3 \pm 8\right.$.stat. $\left.2 \pm 3.9_{\text {sys. }}\right) \mathrm{MeV}$. In the measurements without the constraint, they observed $125.6 \pm 15.7 D_{s}^{+} \rightarrow \tau^{+} v_{\tau}$ signals and further determined $\mathscr{B}\left(D_{s}^{+} \rightarrow \mu^{+} v_{\mu}\right)=\left(0.565 \pm 0.045_{\text {stat. }} \pm\right.$ $\left.0.017_{\text {sys. }}\right) \%$ and $\mathscr{B}\left(D_{s}^{+} \rightarrow \tau^{+} v_{\tau}\right)=\left(6.42 \pm 0.81_{\text {stat. }} \pm 0.18_{\text {sys. }}\right) \%$. Since no significant $D_{s}^{+} \rightarrow e^{+} v_{e}$ signal is observed, they set an upper limit of $\mathscr{B}\left(D_{s}^{+} \rightarrow e^{+} v_{e}\right)<1.2 \times 10^{-4}$ at $90 \%$ C.L..

With the same singly tagged $D_{s}^{-}$samples, they observed $200 \pm 20 D_{s}^{+} \rightarrow \tau^{+}\left(\rho^{+} \bar{v}_{\tau}\right) \nu_{\tau}$ signals and determined $\mathscr{B}\left(D_{s}^{+} \rightarrow \tau^{+} v_{\tau}\right)=\left(5.52 \pm 0.57_{\text {stat. }} \pm 0.21_{\text {sys. }}\right) \% f_{D_{s}^{+}}=\left(257.8 \pm 13.3_{\text {stat. }} \pm 5.2_{\text {sys. }}\right)$ $\mathrm{MeV}$ [24].

Using the three cleanest singly tagged $D_{s}^{-}$hadronic decays $\left(D_{s}^{+} \rightarrow \phi \pi^{-}, K^{* 0} K^{-}\right.$and $\left.K_{S}^{0} K^{-}\right)$, the accumulated $26334 \pm 214$ singly tagged $D_{s}^{-}$mesons. They found $180.6 \pm 15.9 D_{s}^{+} \rightarrow \tau^{+}\left(e^{+} v_{e} \bar{v}_{e}\right) v_{\tau}$ signals and obtained $\mathscr{B}\left(D_{s}^{+} \rightarrow \tau^{+} v_{\tau}\right)=(5.30 \pm 0.47 \pm 0.22) \%$ and $f_{D_{s}^{+}}=(252.5 \pm 11.1 \pm 5.2)$ $\mathrm{MeV}$ [25]. 


\subsection{Measurement of $D_{s}^{+} \rightarrow \ell^{+} v_{\ell}$ at Belle}

In 2013, the Belle collaboration provided an updated analysis of $D_{s}^{+} \rightarrow \ell^{+} v_{\ell}$ with their full dataset of $913 \mathrm{fb}^{-1}$ collected around $\Upsilon(4 S)$ and $\Upsilon(5 S)$ [20]. The analysis used the processes of $e^{+} e^{-} \rightarrow c \bar{c} \rightarrow D_{\text {tag }} K_{\text {frag }} X_{\text {frag }} D_{s}^{*+}, D_{s}^{*+} \rightarrow \gamma D_{s}^{+}$, where $D_{\text {tag }}$ is one of $\bar{D}^{0}$ (reconstructed by $\bar{D}^{0} \rightarrow$ $K^{+} \pi^{-}\left(\pi^{0}\right), K^{+} \pi^{-} \pi^{-} \pi^{+}, K_{S}^{0} \pi^{-} \pi^{-}\left(\pi^{0}\right)$ ), $D^{-}$(reconstructed by $D^{-} \rightarrow K^{+} \pi^{-} \pi^{-}\left(\pi^{0}\right), K_{S}^{0} \pi^{-}\left(\pi^{0}\right)$, $K^{+} K^{-} \pi^{-}$and $\left.K_{S}^{0} \pi^{+} \pi^{-} \pi^{-}\right), \bar{\Lambda}_{c}^{-}$(reconstructed by $\bar{\Lambda}_{c}^{-} \rightarrow \bar{p} K^{+} \pi^{-}\left(\pi^{0}\right), \bar{p} K_{S}^{0}, \bar{\Lambda} \pi^{-}\left(\pi^{0}\right)$ and $\bar{\Lambda} \pi^{-} \pi^{-} \pi^{+}$), $D^{*-}$ or $\bar{D}^{* 0} ; K_{\text {frag }}$ is either $K^{+}$or $K_{S}^{0} ; X$ indicates the possible particle combinations. They accu-

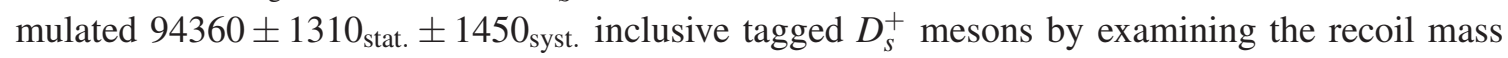
spectra of the $D K X \gamma$ systems. Based on this sample, they observed $492 \pm 26 D_{s}^{+} \rightarrow \mu^{+} v_{\mu}$ signals and $2217 \pm 83 D_{s}^{+} \rightarrow \tau^{+} v_{\tau}$ signals with three $\tau^{+}$decay modes $\tau^{+} \rightarrow e^{+} v_{e} \bar{v}_{e}, \mu^{+} v_{\mu} \bar{v}_{\mu}$ and $\pi^{+} \bar{v}_{\tau}$. They determined $\mathscr{B}\left(D_{s}^{+} \rightarrow \mu^{+} v_{\mu}\right)=\left(0.531 \pm 0.028_{\text {stat. }} \pm 0.020_{\text {sys. }}\right) \%, \mathscr{B}\left(D_{s}^{+} \rightarrow \tau^{+} v_{\tau}\right)=(5.70 \pm$ $0.21_{\text {stat. }}{ }_{-0.30}^{+0.31}$ sys. $) \%$ and $f_{D_{s}^{+}}=\left(255.5 \pm 4.2_{\text {stat. }} \pm 5.1_{\text {sys. }}\right) \mathrm{MeV}$. Since no significant $D_{s}^{+} \rightarrow e^{+} v_{e}$ signal is observed, they set an upper limit of $\mathscr{B}\left(D_{s}^{+} \rightarrow e^{+} v_{e}\right)<1.0(0.83) \times 10^{-4}$ at $95 \%(90 \%)$ C.L..

\subsection{Measurement of $D_{s}^{+} \rightarrow \ell^{+} v_{\ell}$ at BaBar}

Using the same method as used by Belle, the BaBar collaboration presented an analysis of $D_{s}^{+} \rightarrow \ell^{+} v_{\ell}$ by analyzing $521 \mathrm{fb}^{-1}$ of data taken near $10.58 \mathrm{GeV}$ [21]. The analysis also used the processes of $e^{+} e^{-} \rightarrow c \bar{c} \rightarrow D_{\mathrm{tag}} K_{\mathrm{frag}} X_{\mathrm{frag}} D_{s}^{*+}, D_{s}^{*+} \rightarrow \gamma D_{s}^{+}$, where $D_{\mathrm{tag}}$ is one of $\bar{D}^{0}$ (reconstructed by $\bar{D}^{0} \rightarrow K^{+} \pi^{-}\left(\pi^{0}\right), K^{+} \pi^{-} \pi^{-} \pi^{+}\left(\pi^{0}\right), K_{S}^{0} \pi^{-} \pi^{-}\left(\pi^{0}\right)$ ), $D^{-}$(reconstructed by $D^{-} \rightarrow K^{+} \pi^{-} \pi^{-}\left(\pi^{0}\right)$, $K_{S}^{0} \pi^{-}\left(\pi^{0}\right)$ and $\left.K_{S}^{0} \pi^{+} \pi^{-} \pi^{-}\right), \bar{\Lambda}_{c}^{-}$(reconstructed by $\bar{\Lambda}_{c}^{-} \rightarrow \bar{p} K^{+} \pi^{-}\left(\pi^{0}\right), \bar{p} K_{S}^{0}$ and $\bar{p} K_{S}^{0} \pi^{-} \pi^{+}$), They obtained $(67.2 \pm 1.5) \times 10^{3}$ inclusive $D_{s}^{+}$mesons. From this sample, they observed $275 \pm 17$, $408 \pm 42$ and $340 \pm 32$ signals of $D_{s}^{+} \rightarrow \mu^{+} v_{\mu} D_{s}^{+} \rightarrow \tau^{+}\left(v_{e} \bar{v}_{e}\right) v_{\tau}$ and $D_{s}^{+} \rightarrow \tau^{+}\left(v_{\mu} \bar{v}_{\mu}\right) v_{\tau}$. The decay branching fractions are measured to be $\mathscr{B}\left(D_{s}^{+} \rightarrow \mu^{+} v\right)=\left(0.602 \pm 0.038_{\text {stat. }} \pm 0.034_{\text {sys. }}\right) \%$, $\mathscr{B}\left(D_{s}^{+} \rightarrow \tau^{+} v\right)=\left(5.07 \pm 0.52_{\text {stat. }} \pm 0.68_{\text {sys. }}\right) \%$ and $\mathscr{B}\left(D_{s}^{+} \rightarrow \tau^{+} v\right)=\left(4.91 \pm 0.47_{\text {stat. }} \pm 0.54_{\text {sys. }}\right) \%$, respectively, The decay constant is determined to be of $f_{D_{s}^{+}}=\left(258.6 \pm 6.4_{\text {stat. }} \pm 7.5_{\text {sys. }}\right) \mathrm{MeV}$. Since no significant $D_{s}^{+} \rightarrow e^{+} v_{e}$ signal is observed, they set an upper limit of $\mathscr{B}\left(D_{s}^{+} \rightarrow e^{+} v_{e}\right)<$ $2.3 \times 10^{-4}$ at $90 \%$ C.L..

\subsection{Measurement of $D_{s}^{+} \rightarrow \ell^{+} v_{\ell}$ at BESIII}

From a dataset of $0.482 \mathrm{fb}^{-1}$ collected at $4.009 \mathrm{GeV}$ with the BESIII detector in 2011, $15127 \pm$ 312 singly tagged $D_{s}^{-}$mesons are accumulated from 9 hadronic decays $K_{S}^{0} K^{-}, K^{+} K^{-} \pi^{-}, K^{+} K^{-} \pi^{-} \pi^{0}$, $K_{S}^{0} K^{+} \pi^{-} \pi^{-}, \pi^{+} \pi^{-} \pi^{-}, \pi^{-} \eta, \pi^{-} \eta_{\pi^{+} \pi^{-} \eta}^{\prime}, \pi^{-} \eta_{\gamma \rho^{0}}^{\prime}$ and $\rho^{-} \eta$, with the fitted $M_{\mathrm{BC}}$ spectra shown in Fig. 3 (Left). At the recoil sides of these tagged $D_{s}^{-}$mesons, the candidates for $D^{+} \rightarrow \ell^{+} v_{\ell}$ are selected. Figure 3 (right) shows the fit to the $M_{\text {miss }}^{2}$ distribution of the accepted candidates. In the fits, the ratio of the $D_{s}^{+} \rightarrow \mu^{+} v_{\mu}$ and $D_{s}^{+} \rightarrow \tau^{+} v_{\tau}$ yields has been constrained by the SM prediction. BESIII obtain $69.3 \pm 9.3$ signals of $D_{s}^{+} \rightarrow \mu^{+} v_{\mu}$ or $32.5 \pm 4.3$ signals of $D_{s}^{+} \rightarrow \tau^{+} v_{\tau}$. These give the absolute branching fractions to be $\mathscr{B}\left(D_{s}^{+} \rightarrow \mu^{+} v_{\mu}\right)=\left(0.495 \pm 0.067_{\text {stat. }} \pm 0.026_{\text {sys. }}\right) \%$ and $\mathscr{B}\left(D_{s}^{+} \rightarrow \tau^{+} v_{\tau}\right)=\left(4.83 \pm 0.65_{\text {stat. }} \pm 0.26_{\text {sys. }}\right) \%$. Here, the measured $\mathscr{B}\left(D_{s}^{+} \rightarrow \mu^{+} v_{\mu}\right)$ has been corrected by $1 \%$ to considering the $\gamma \mu^{+} v_{\mu}$ final state. Using the measured $B\left(D_{s}^{+} \rightarrow \ell^{+} v_{\ell}\right)$ and the $\left|V_{u d}\right|$ from the SM global fit [1], the $D_{s}^{+}$decay constant is determined to be $f_{D_{s}^{+}}=241.0 \pm 16.3_{\text {stat. }} \pm$ 6.6 $6_{\text {sys. }} \mathrm{MeV}[26]$. 

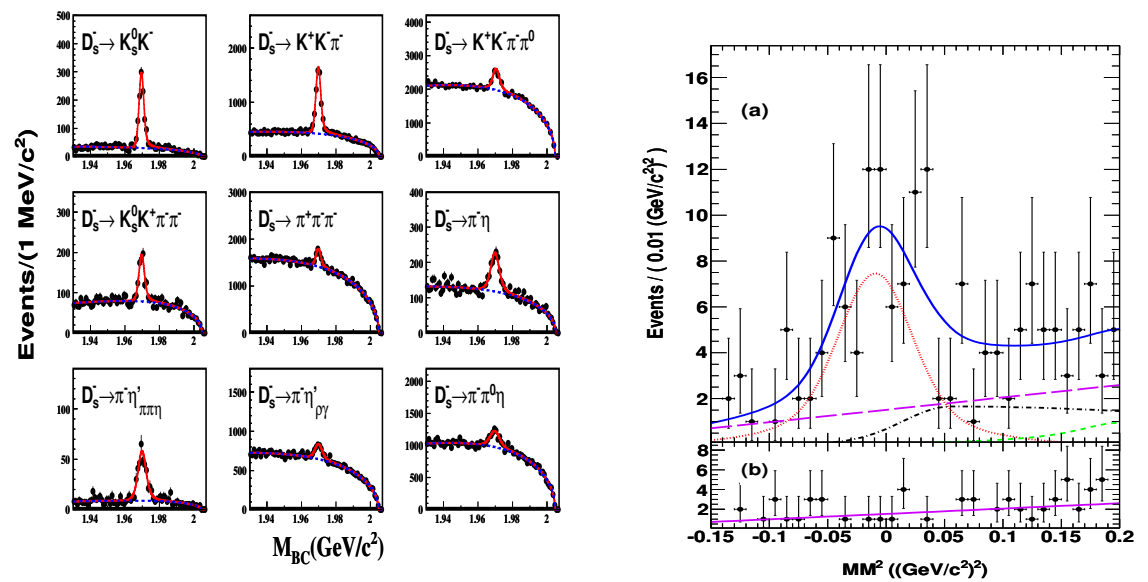

Figure 3: (Left) Fits to the $M_{\mathrm{BC}}$ spectra for the singly tagged $D_{s}^{-}$candidates. (Right) Simultaneous fits to the $M_{\text {miss }}^{2}$ distribution of the $D_{s}^{+} \rightarrow \ell^{+} v_{\ell}$ candidates. The error bars are data. The red curves are the best fits. The red dotted curve is the $D_{s}^{+} \rightarrow \mu^{+} v_{\mu}$ signal shape. The black dot-dashed curve is the $D_{s}^{+} \rightarrow \tau^{+} v_{\tau}$ signal shape. The purple curve is the non- $D_{s}^{+}$background. The green curve is the $D_{s}^{+}$backgrounds.

\section{Summary and discussions}

In recent ten years, much progress in the experimental studies $D_{(s)}^{+} \rightarrow \ell^{+} v_{\ell}(\ell=e, \mu$ or $\tau)$ has been reached in the CLEO-c, Belle, BaBar and BESIII experiments. Ref. [1] has well reviewed these measurements. The averages of $f_{D^{+}}\left|V_{c d}\right|$ and $f_{D_{s}^{+}}\left|V_{c s}\right|$ are $45.91 \pm 1.05$ and $250.9 \pm 4.0 \mathrm{MeV}$, respectively. Further, average of $\left|V_{c d}\right|$ and $\left|V_{c s}\right|$ are discussed.

- Average of $\left|V_{c d}\right|$ : Using $f_{D^{+}}=209.2 \pm 3.3 \mathrm{MeV}$ calculated by LQCD [27] and the average of measurements of $D^{+} \rightarrow \ell^{+} v_{\ell}$ at CLEO-c [10] and BESIII [11], one obtains $\left|V_{c S}\right|=$ $0.219 \pm 0.005 \pm 0.003$, where the first uncertainty, which is dominant, is experimental, the second is theoretical. Using $f_{+}^{\pi}(0)=0.666 \pm 0.029$ calculated by LQCD [27] and the average of measurements of $D \rightarrow \pi \ell^{+} v_{\ell}$ decays at Belle [28], CLEO-c [29], BaBar [30] and BESIII [31], one obtains $\left|V_{c d}\right|=0.214 \pm 0.003 \pm 0.009$, where the first uncertainty is experimental, the second, which is dominant, is from the theoretical uncertainty of the form factor. Combining these two determinations with the one measured in neutrino scattering data $\left|V_{c d}\right|=0.230 \pm 0.011$, one obtains $\left|V_{c d}\right|=0.220 \pm 0.005$ [1].

- Average of $\left|V_{c s}\right|$ : Combining $f_{D_{s}^{+}}=(248.6 \pm 2.7) \mathrm{MeV}$ calculated by LQCD [4] and the measurements of $D_{s}^{+} \rightarrow \ell^{+} v_{\ell}$ at Belle [20], CLEO-c [23, 24, 25] and BaBar [21], one obtains $\left|V_{c s}\right|=1.008 \pm 0.021$, where the dominated uncertainty is from experiments. Using $f_{+}^{K}(0)=$ $0.747 \pm 0.019$ calculated by LQCD [32] and the average of measurements of $D \rightarrow K(\pi) \ell^{+} v_{\ell}$ at Belle [28], CLEO-c [29], BaBar [33] and BESIII [31], one obtains $\left|V_{c s}\right|=0.975 \pm 0.007 \pm$ 0.025 , where the first uncertainty is experimental and the second, which is dominant, is from the theoretical uncertainty of the form factor. These two kinds of measurements lead to $\left|V_{c s}\right|=0.995 \pm 0.016[1]$. 
At present, the averages of the measured decay constants $f_{D^{+}}$and $f_{D_{s}^{+}}$are $203.8 \pm 4.6 \pm$ 1.0 [35] and 258.9 $\pm 3.8 \pm 1.8$ [34], respectively. They are consistent with the most recent and precise LQCD calculations $f_{D^{+}}=212.6 \pm 0.4_{-1.2}^{+1.0}$ and $f_{D_{s}^{+}}=249.0 \pm 0.3_{-1.5}^{+1.1}$ [3] within about $1.8 \sigma$ and 2.2 $\sigma$. In addition, a recent global analysis reported in Refs. [34, 35] gives unitarity tests $\left|V_{u d}\right|^{2}+\left|V_{c d}\right|^{2}+\left|V_{t d}\right|^{2}=0.996 \pm 0.002$ and $\left|V_{c d}\right|^{2}+\left|V_{c s}\right|^{2}+\left|V_{c b}\right|^{2}=1.015 \pm 0.022$, where the previous one deviates from unit by $2 \sigma$ and the error of the latter one is dominated by the uncertainty of $\left|V_{c s}\right|$.

This proceeding also reports two recent BESIII results of $D^{+} \rightarrow \tau^{+} v_{\tau}$ and $D_{s}^{+} \rightarrow \ell^{+} v_{\ell}$, which have not been included in the above averages. However, the weights of these two measurements should be small.

In 2016, BESIII collected about $3 \mathrm{fb}^{-1}$ data taken near $\sqrt{s}=4.18 \mathrm{GeV}$. Further improved measurements of $\mathscr{B}\left(D_{s}^{+} \rightarrow \ell^{+} v_{\ell}\right), f_{D_{s}^{+}}$and $\left|V_{c s}\right|$ are expected in the near future. In the foreseen future, more precise measurements of $D_{(s)}^{+} \rightarrow \ell^{+} v_{\ell}$ with larger $D_{(s)}^{+}$samples at BESIII, BelleII or $\mathrm{LHCb}$ (upgraded ara) will further benefit the tests on the LQCD calculations of $f_{D_{(s)}^{+}}$and the CKM unitarity.

\section{References}

[1] C. Patrignani et al. (Particle Data Group), Chin. Phys. C 40, 100001 (2016).

[2] E. Follana et al., (HPQCD and UKQCD Collaborations), Phys. Rev. Lett. 100, 062002 (2008).

[3] A. Bazavov, et al. (Fermilab Lattice and MILC Collaborations), Phys. Rev. D 90, 074509 (2010).

[4] S. Aoki et al., Eur. Phys. J. C 74, 2890 (2014).

[5] J. Adler et al. (MARKIII Collaboration), Phys. Rev. Lett. 60, 1375 (1998).

[6] J. Z. Bai et al. (BES Collaboration), Phys. Lett. B 429, 188 (1998).

[7] M. Ablikim et al. (BES Collaboration), Phys. Lett. B 610, 183 (2005).

[8] G. Bonvicini et al. (CLEO Collaboration), Phys. Rev. D 70, 112004 (2004).

[9] M. Artuso et al. (CLEO Collaboration), Phys. Rev. Lett. 95, 251801 (2005).

[10] B.I. Eisenstein et al. (CLEO Collaboration), Phys. Rev. D 78, 052003 (2008).

[11] M. Ablikim et al. (BESIII Collaboration), Phys. Rev. D 89, 051104 (2014).

[12] S. Aoki et al. (WA75 Collaboration), Prog. Theor. Phys. 89, 131 (1993).

[13] K. Kodama et al. (E653 Collaboration), Phys. Lett. B 382, 299 (1996).

[14] Y. Alexandrov et al. (BEATRICE Collaboration), Phys. Lett. B 478, 31 (2000).

[15] M. Acciarri et al. (L3 Collaboration), Phys. Lett. B 396, 327 (1997)

[16] G. Abbiendi et al. (OPAL Collaboration), Phys. Lett. B 516, 236 (2001)

[17] R. Barate et al. (ALEPH Collaboration), Phys. Lett. B 528, 1 (2002)

[18] M. Chada et al. (CLEO Collaboration), Phys. Rev. D 58, 032002 (1998).

[19] L. Widhalm et al. (Belle Collaboration), Phys. Rev. Lett. 100, 241801 (2008).

[20] A. Zupanc et al. (Belle Collaboration), JHEP 1309, 139 (2013). 
[21] P. del Amo Sanchez et al. (BaBar Collaboration), Phys. Rev. D 82, 091103(R) (2010).

[22] J. Z. Bai et al. (BES Collaboration), Phys. Rev. Lett. 74, 4599 (1995).

[23] J.P. Alexander et al. (CLEO Collaboration), Phys. Rev. D 79, 052001 (2009).

[24] P. Naik et al. (CLEO Collaboration), Phys. Rev. D 80, 112004 (2009).

[25] P.U.E. Onyisi et al. (CLEO Collaboration), Phys. Rev. D 79, 052002 (2009).

[26] M. Ablikim et al. (BESIII Collaboration), Phys. Rev. D 94, 072004 (2016).

[27] H. Na, et al. (HPQCD Collaboration), Phys. Rev. D 84, 114505 (2011).

[28] L. Widhalm et al. (Belle Collaboration), Phys. Rev. Lett. 97, 061804 (2006).

[29] D. Besson et al. (CLEO Collaboration), Phys. Rev. D80, 032005 (2009).

[30] J. P. Lees et al. (BaBar Collaboration), Phys. Rev. D91, 052022 (2015).

[31] M. Ablikim et al. (BESIII Collaboration), Phys. Rev. D 97, 072012 (2015).

[32] H. Na, et al. (HPQCD Collaboration), Phys. Rev. D 82, 114506 (2010).

[33] B. Aubert et al. (BaBar Collaboration), Phys. Rev. D 76, 052005 (2007).

[34] Y. Fang, G. Rong, H. L. Ma, J. Y. Zhao, Eur. Phys. J. C 75, 10 (2015).

[35] G. Rong, Y. Fang, H. L. Ma, J. Y. Zhao, Phys. Lett. B 743, 315 (2015). 